\title{
Formulation and In vitro Evaluation of Unfolding Type Expandable Gastroretentive Film of Enalapril Maleate
}

\author{
Md. Bashir Ullah ${ }^{1}$, Md. Rezaul Karim¹, Md. Shamsul Alam², Md. Rajib Hassan", \\ Mohiuddin Ahmed Bhuiyan ${ }^{1}$ and Md. Sohel Rana ${ }^{2}$ \\ ${ }^{1}$ Pharmaceutical Technology Research Laboratory, Department of Pharmacy, University of Asia Pacific, \\ 74/A, Green Road, Farmgate Dhaka-1215, Bangladesh \\ ${ }^{2}$ Department of Pharmacy, Jahangirnagar University, Savar, Dhaka-1342, Bangladesh
}

Received: March 12, 2017; Accepted: March 30, 2017; Published (Web): July 31, 2017

\begin{abstract}
The present work was based on the development and characterization of unfolding type gastroretentive dosage form appropriate for controlled release of enalapril maleate. Drug loaded films were prepared by solid dispersion technique using methocel K15 and eudragit RSPO and eudragit RLPO as polymers and polyethylene glycol 400 (PEG 400) as the plasticizer. The film folded in a capsule shell was shown to unfold in the gastric juice and provide drug release up to $12 \mathrm{~h}$ in the acidic medium. Formulations provided satisfactory unfolding characteristics allowing expansion to remain in the stomach. Formulation containing above $60 \%$ content of eudragit RSPO and eudgrait RLPO combination of total polymer content provided satisfactory film integrity over 12 hours. The result revealed that formulation F1 showed a minimum percentage of drug release of $63.41 \%$ followed by formulation F2, F3, F4 and F5 with 66.76\%, $80.21 \%$, 83.26\% and $86.92 \%$ release in 8 hour respectively. Formulation with high proportion of eudragit RLPO and RSPO combination in total polymer content was found to be slow in drug release and lower the release from the polymeric film over time. As the concentration of HPMC K 15 in total polymer content increased the release rate of enalapril maleate as well as \% release from the polymeric film also increased over time. Most of the formulation followed Higuchi release kinetics followed by Korsmeyer release kinetics. The drug release mechanism from the film follows Fickian and Non Fickian release kinetics. The films were evaluated for mechanical properties, in vitro drug release and unfolding behavior based on the mechanical shape memory of polymers. Absence of drug polymer interaction and uniform drug dispersion in the polymeric layers was revealed by DSC, FT-IR and SEM studies.
\end{abstract}

Key words: Gastroretention, Unfolding type, Enalapril maleate, Polymer.

\section{Introduction}

Oral controlled release dosage forms have been developed over the past three decades due to their considerable therapeutic advantages such as ease of administration, patient compliance and flexibility in formulation. Furthermore, the relatively brief gastric emptying time in humans which normally an average 2-3 $\mathrm{h}$ through the major absorption zone, i.e., stomach and upper part of the intestine can result in incomplete drug release from the drug delivery system leading to reduced efficacy of the administered dose (Rouge et al., 1996). Therefore, control of placement of a drug delivery system in a specific region of the gastro intestinal tract (GIT) offers advantages for a variety of important drugs characterized by a narrow absorption window in the GIT or drugs with a stability problem (Singh and Kim, 2000). These considerations have led to the development of a unique oral controlled release dosage form with gastroretentive properties. After oral administration, such a dosage form would be retained in the stomach and release the drug in a controlled and prolonged manner, so that the drug could be supplied continuously to its absorption sites in the upper gastrointestinal tract (Streubel et al., 2006). Gastroretentive dosage form (GRDF) can

Correspondence to: Mohiuddin Ahmed Bhuiyan; E-mail: mohiuddin@uap-bd.edu 
remain in the gastric region for several hours and hence significantly prolong the gastric residence time of drugs. Prolonged gastric retention improves bioavailability, reduces drug waste, and improves solubility of drugs that are less soluble in a high $\mathrm{pH}$ environment. It is also suitable for local drug delivery to the stomach and proximal small intestines (Ali $e t$ al., 2005). Gastroretention helps to provide better availability of new products with suitable therapeutic activity and substantial benefits for patients. This mode of administration would achieve the known pharmacokinetic and pharmacodynamic advantages of controlled release dosage form of these drugs. The need for GRDFs has led to extensive efforts in both academia and industry towards the development of such drug delivery systems. Several approaches have been proposed to retain the dosage forms in the stomach. These methods include bioadhesive system, swelling system and expanding system and floating system. In fact the buoyant dosage unit enhances gastric residence time without affecting the intrinsic rate of gastric emptying (Banker and Anderson, 2007).

The expandable GRDFs are usually based on configuration which enables convenient oral intake; expanded form that is achieved in the stomach and thus prevents passage through the pyloric sphincter; and finally another small form that is achieved in the stomach when retention is no longer required i.e. after the GRDF has released its active ingredient, thereby enabling evacuation. The expansion can be achieved by swelling or by unfolding in the stomach (Klausner et al., 2003).

Unfolding systems might represent one of the best alternatives to resist to the gastric emptying. Indeed the unfolding system is usually introduced through a hard gelatin capsule for an easier intake by the patient. In the stomach the carrier is dissolved due to the action of gastric acid, releasing the GRDF system which unfolds or opens out to achieve its extended configuration. The carrier should maintain unfolded properties for extended time spans. The functionality of these systems is based on dimensions and mechanical shape memory (Kagan et al., 2006).
Enalapril maleate is the maleate salt form of enalapril, a dicarbocyl-containing peptide and angiotensin converting enzyme (ACE) inhibitor with antihypertensive activity. It is chemically described as (S)-1[N-[1-(ethoxycarbonyl)-3-phenylpropyl]-Lalanyl]-L-proline, (Z)-2-butenedioate salt (1:1). It is a prodrug which when administered orally is hydrolyzed to release the active ACE inhibitor enalaprilat. Enalapril maleate is $60 \%$ absorbed and $40 \%$ bioavailable as enalaprilat. Both compounds undergo renal excretion without further metabolism. Inhibition of enalapril is associated with reductions in plasma angiotensin II and plasma aldosterone, and with increases in plasma renin activity and plasma angiotensin I (Davies et al., 1984). ACE is a peptidyl dipeptidase that catalyzes the conversion of angiotensin I to the vasoconstrictor substance, angiotensin II. The beneficial effects of enalapril in hypertension and heart failure appear to result primarily from suppression of the reninangiotensin aldosterone system. Inhibition of ACE results in decreased plasma angiotensin II, which leads to decreased blood pressure and aldosterone secretion (Hardman et al., 2001). Enalapril is used in the management of mild to severe hypertension. The drug has been used as monotherapy or in combination with other classes of antihypertensive agents. Enalaprilat is used in the management of hypertension when oral therapy is not practical (McEvoy, 2003). It is also indicated for the treatment of hypertension and as adjunctive therapy in the management of heart failure, in patients who are not responding adequately to diruretics and digitalis (Hussar, 1992).

The purpose of this research was to develop a novel expandable GRDF, based on unfolding mechanism. Dosage form size superior to pylorus diameter $12.8 \pm 7.0 \mathrm{~mm}$ during the gastric emptying to allow retention of the GRDF in stomach gets sustained release action and this group is comprised of expandable and unfolding system. The polymers used in such systems are able to significantly increase the dimensions of the dosage form, in order to be larger than the pylorus diameter. GRDF consists of a drug loaded polymeric film, folded into a hard gelatin 
capsule. Gastric retention is achieved due to unfolding of the dosage form within 15-20 min. Enalapril maleate is selected as the drug candidate for this work because of its comparatively short biological half life in order to retain in upper part of intestine for sustained release in stomach. Lower pKa value and its lipid solubility also allow good absorption in acidic media of stomach.

\section{Methodology}

Materials: Enalapril maleate was a kind gift from SK+F Bangladesh Ltd. HPMC (methocel K15) was obtained from Colorcon Asia Pvt Ltd. Eudragit RSPO and eudragit RLPO were gifted by Evonik Industries AG, Mohakhali, Dhaka. All other reagents and chemicals were of suitable analytical grade and were used as received.

Formulation design: Total five batches were formulated and designed as F1 to F5 as shown in Table 1. All the formulations were prepared by using same polymer and excipient. Only the variable was the amount of methocel K15 and eudragit RSPO and eudragit RLPO (1:1) combination used in the formulations. Drug loading and other excipients were same. The rate reterdant methocel $\mathrm{K} 15$ as highly swellable polymer allow expansion satisfactory in gastric environment and eudragit retains film integrity satisfactory over time.

Preparation of polymeric film of enalapril maleate: The polymeric film was prepared by solvent evaporation method. A polymeric dispersion of HPMC K 15, eudragit RSPO and eudragit RLPO was prepared by dissolving in optimum quantity of ethanol and dichloromethane respectively. Ethanol and dichloromethane used this experiment as 1:1 ratio. Then the measured quantity of enalapril maleate was dissolved and added to the polymeric mixer. Finally required quantity PEG 400 was added to the dispersion as a plasticizer. Then the dispersion was stirred vigorously to get uniform distribution. The resulting dispersion was then poured into petridish $(120 \mathrm{~mm})$ and allowed to dry at room temperature for 24 hours. The film obtained as a result of solvent evaporation was carefully removed and had an area of $110 \mathrm{~cm}^{2}$ which was cut into $4 \mathrm{~cm}$ $\times 2 \mathrm{~cm}=8 \mathrm{~cm}^{2}$ rectangle units.

The amount of enalapril maleate was calculated in this experiment for $110 \mathrm{~cm}^{2}$ film area where per (unit) $8 \mathrm{~cm}^{2}$ film containes $20 \mathrm{mg}$ of enalapril maleate and had an average weight of $137 \mathrm{mg}$. The prepared film was folded into zigzag pattern and then inserted into 00 size capsule shell (Figure 1).

Table 1. Formulation ingredients of enalapril maleate loaded polymeric film.

\begin{tabular}{cccccc}
\hline Formulations & $\begin{array}{c}\text { Enalapril maleate } \\
(\mathrm{mg})\end{array}$ & $\begin{array}{c}\text { Methocel K 15 } \\
\text { M (mg) }\end{array}$ & $\begin{array}{c}\text { Eudragit } \\
\text { RSPO (mg) }\end{array}$ & $\begin{array}{c}\text { Eudragit RLPO } \\
(\mathrm{mg})\end{array}$ & $\begin{array}{c}\text { PEG 400 } \\
(\mathrm{ml})\end{array}$ \\
\hline F 1 & 280 & 360 & 420 & 420 & 0.5 \\
F 2 & 280 & 420 & 390 & 390 & 0.5 \\
F 3 & 280 & 480 & 360 & 360 & 0.5 \\
F 4 & 280 & 540 & 330 & 330 & 0.5 \\
F 5 & 280 & 600 & 300 & 300 & \\
\\
\end{tabular}

Figure 1. (A) Film of enalapril maleate, (B) Zigzag folding pattern, (C) Film inserted into ‘00’ size capsule shell. 
Unfolding behavior of GRDFs in vitro: The prepared polymeric film was folded in a zigzag manner and inserted into 00 size capsule. In vitro unfolding study was carried out in a beaker of $500 \mathrm{ml}$ of $0.1 \mathrm{~N} \mathrm{HCl}(\mathrm{pH} \mathrm{1.2)}$ at constant temperature. The drug loaded folded film was placed in the beaker and the films were examined for their unfolding behavior at several time intervals.

In vitro drug release study: In vitro drug release study of various batches were conducted in simulated gastric fluid medium prepared (pH 1.2, $900 \mathrm{ml}$ ) using USP apparatus I (USP (XXII) dissolution apparatus, Electro VDA-8DR, India) with constant temperature maintained at $37 \pm 0.5^{\circ} \mathrm{C}$ and $50 \mathrm{rpm}$ till $8 \mathrm{~h}$. An aliquot of $5 \mathrm{ml}$ was withdrawn and replaced with another $5 \mathrm{ml}$ of fresh simulated gastric fluid medium at various time intervals. The content of enalapril maleate in sample was analyzed by double beam UVVis Spectrophotometer (UV mini-1240, Shimadzu Corporation, Japan) at $209 \mathrm{~nm}$. The corresponding concentrations were determined from the standard curve of enalapril maleate prepared in simulated gastric fluid medium at $209 \mathrm{~nm}$.

\section{Characterization of Polymeric Film of Enalapril Maleate}

Differential Scanning Calorimetry: Thermal analysis of drug-excipient compatibility was studied by Differential Scanning Calorimeter (DSC, Shimadzu Corporation, Japan).The DSC thermogram of pure drug enalapril maleate, solid dispersion of physical mixture (HPMC+ eudragit RSPO + eudragit $\mathrm{RLPO}+$ drug ), and polymeric film was estimated in terms of their endotherms using DSC. The samples were heated in temperature range from 30 to $300^{\circ} \mathrm{C}$ at a heating rate of $10^{\circ} \mathrm{C}$ per minute under nitrogen atmosphere.

Scanning Electron Microscopy: The morphology of the polymeric film was studied by scanning electron microscope (SEM). The film was examined in a JSM-6490LA electron microscope (Jeol, Tokyo, Japan) equipped with an EM-ASID 11 Scanning Image Observation Device using secondary electron imaging at an acceleration voltage of $20 \mathrm{kV}$ at different magnification.

Fourier Transform Infrared (FT-IR) Spectroscopy: FT-IR is particularly useful for identification of organic molecular groups and compounds due to the range of functional groups, side chains and cross-links involved, all of which will have characteristic vibrational frequencies in the infrared range (4000- $400 \mathrm{~cm}^{-1}$ ). The FTIR of pure drug enalapril maleate, physical mixure (HPMC + eudragit RSPO + eudragit RLPO + drug ), and polymeric film was estimated in term of their vibrational frequencies in the infra-red range (4000$400 \mathrm{~cm}^{-1}$ ) using FT-IR 8400S Shimadzu spectrophotometer.

\section{Result and Discussion}

Unfolding behavior of the film and film integrity: Reduction in mechanical shape after prolonged stress applied was the major difficulty in unfolding type of polymeric films. Polymeric material with glass transition temperatures close to ambient temperature with its resiliency to restore its original shape were therefore useful in polymeric formulation since they undergoes less plastic deformation and retained their elasticity even when they were folded (Verma et al., 2014).

GRDFs prepared by zigzag methods were evaluated for their in vitro unfolding behavior. The GRDFs of zigzag manner were unfolded within 1530 min (Figure 2). Apart from folding pattern, for proper unfolding of a film, mechanical shape memory (resiliency to restore its original shape) is required. Such shape memory polymers may have the glass transition (Tg) at about room temperature.

Film integrity of the formulations (F1- F5) primarily depends on the concentration of eudragit polymer as the water insoluble polymer. Formulations containing above $60 \%$ content of eudragit RSPO and eudragit RLPO combination of total polymer content provided satisfactory film integrity over 12 hours. Formulation F1 was found to have higher integrity followed by F2, F3, F4 and F5 
respectively as the eudragit content also reduced respectively. As HPMC $\mathrm{K} 15$ is water soluble polymer, film integrity does not depend on HPMC K 15 content. Polyethylene glycol 400 is the favorable plasticizer to impart elasticity of the polymeric film to allow unfolds satisfactory in the dissolution medium.

In vitro dissolution study: The in vitro drug release study from all five set of formulation (F1, F2, F3, F4 and F5) was performed. The result revealed that formulation F1 showed a minimum percentage of drug release of $63.41 \%$ followed by the formulation F2, F3, F4 and F5 of $66.76 \%, 80.21 \%, 83.26 \%$ and $86.92 \%$ in 8 hours respectively. Formulation with high proportion of eudragit RLPO and eudragit RSPO combination in total polymer content was found to be slow release rate of drug from the polymeric film over time. As the concentration of HPMC K 15 in total polymer content increases the release rate of enalapril maleate from the polymeric film also increase over time.
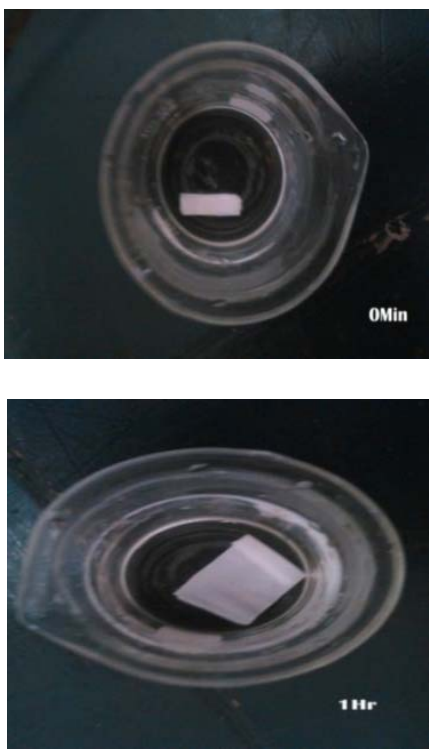
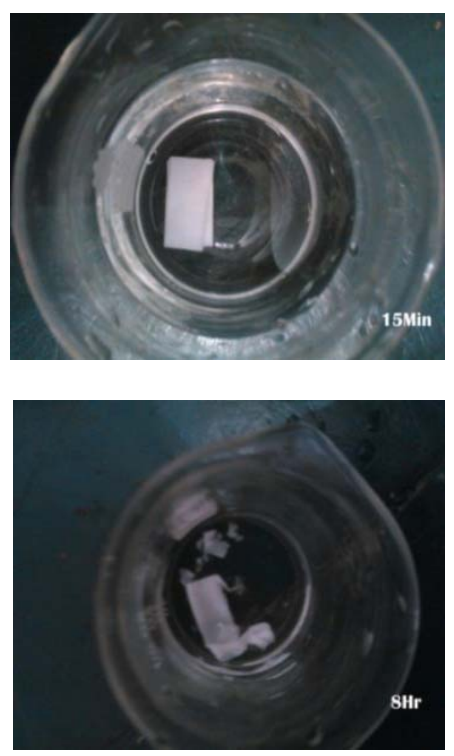
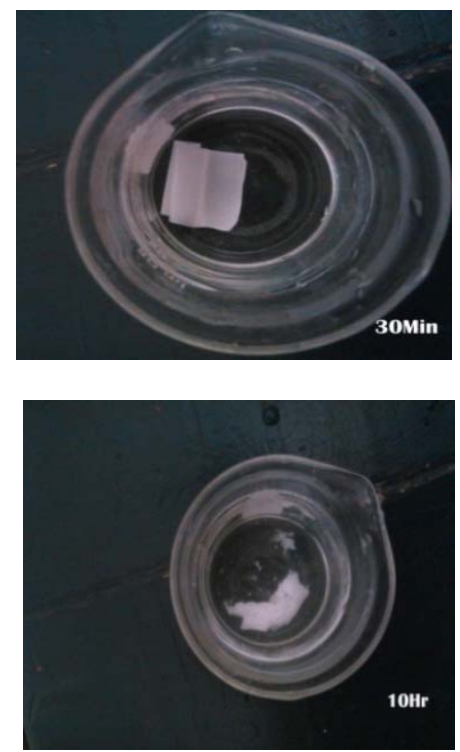

Figure 2. Unfolding behavior of enalapril maleate film at time $0 \mathrm{~min}, 15 \mathrm{~min}, 30 \mathrm{~min}$, 1hour, 8 hours and 10 hours respectively.

\section{Drug Release Kinetics}

To explore the kinetic behavior, results of in vitro release of enalapril maleate from polymeric film was fitted to zero order, first order, Higuchi square root equation Koresmeyer-Peppas and HixsonCrowell equation (Figure 3) and the value of $k$ was determined for different models.

The dissolution data of F1 best fits with Higuchi kinetic model as the value of $\mathrm{R}^{2}$ is highest (0.976) and this is similar also in case of F2, F3 and F4 (Table 2). F5 best fits with first order kinetic model. The values of release component (n) obtained from Korsmeyer-Peppas model indicate the release pattern of enalapril maleate from F1 and F2 is followed by
Fickian release pattern, more specifically diffusion controlled release mechanism which means that zero order release rate was changed over time. On the other hand, the release pattern of enalapril maleate from F3, F4 and F5 is followed by anomalous transport (non Fickian release kinetics). Anomalous transport mechanism refers to coupling of both diffusion and erosion controlled drug release.

\section{Characterization of the film}

Differential Scanning Calorimetry (DSC): DSC study of drug -polymer mixture (solid dispersion), pure drug (enalapril maleate) and GRDF (film) of batch F3 showed the thermogram at Figure 4. 

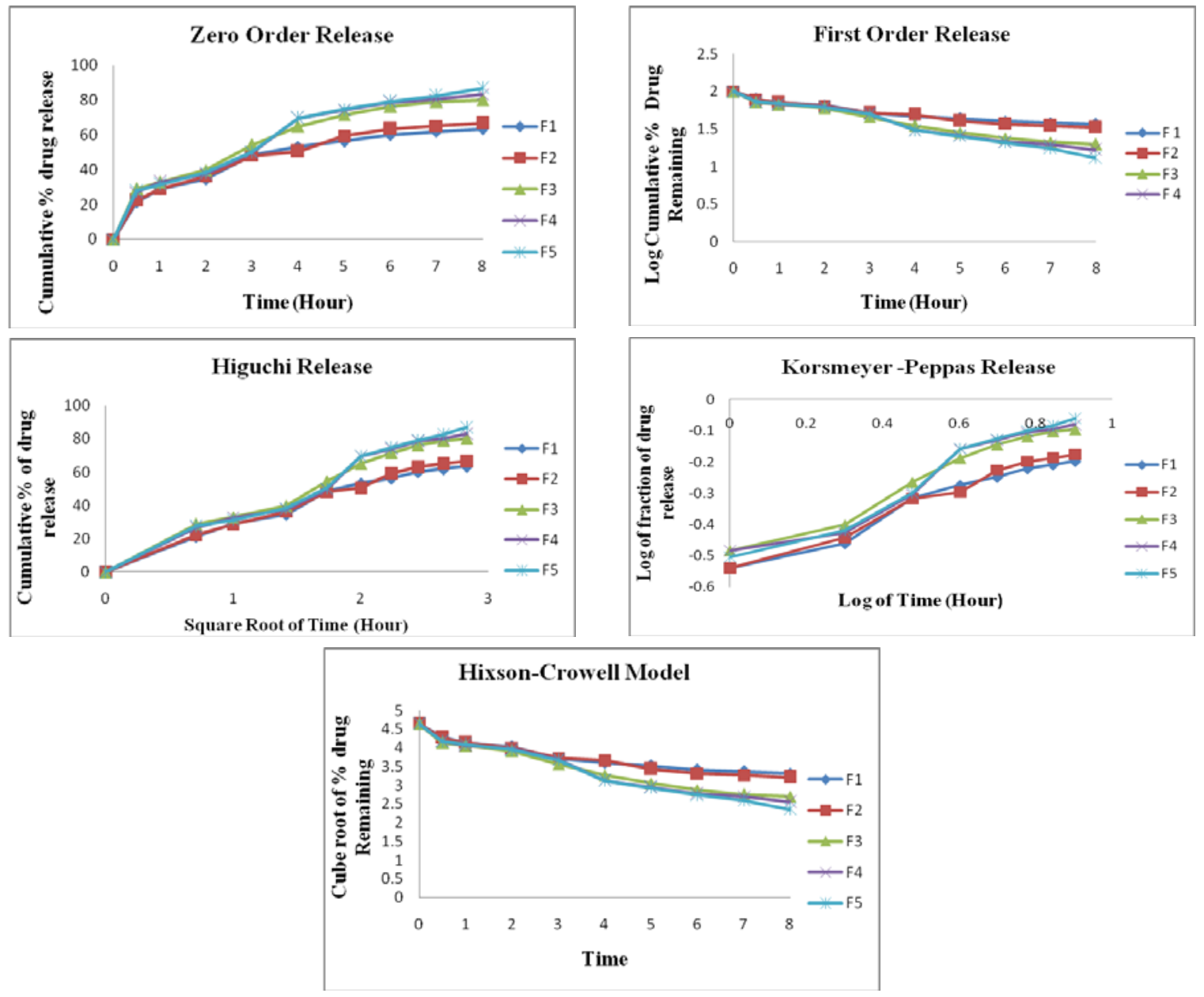

Figure 3. A) Zero order, B) First order, C) Higuchi, D) Korsemeyer-Peppas and E) Hixon-Crowell release kinetics of enalapril maleate from the polymeric film with different concentration of methocel K15M and eudragit RSPO and eudragit RLPO combination.

\section{Interpretation of Release Rate Constant and $\mathbf{R}^{2}$ Values for Different Release Kinetics}

Table 2. $\mathbf{R}^{2}$ values and release rate constant of formulations.

\begin{tabular}{llllllllllll}
\hline \multirow{2}{*}{ Sl. } & \multicolumn{2}{c}{ Zero Order } & \multicolumn{2}{c}{ First Order } & \multicolumn{2}{c}{ Higuchi } & \multicolumn{3}{c}{ Korsmeyer -Peppas } & \multicolumn{2}{c}{ Hixson-Crowell } \\
\cline { 2 - 11 } & $\mathrm{R}^{2}$ & $\mathrm{~K}_{0}$ & $\mathrm{R}^{2}$ & $\mathrm{~K}_{1}$ & $\mathrm{R}^{2}$ & $\mathrm{~K}_{\mathrm{H}}$ & $\mathrm{R}^{2}$ & $\mathrm{n}$ & $\mathrm{K}_{\mathrm{K}}$ & $\mathrm{R}^{2}$ & $\mathrm{~K}_{\mathrm{HC}}$ \\
\hline F1 & 0.842 & 6.858 & 0.923 & -0.117 & $\mathbf{0 . 9 7 6}$ & 22.65 & 0.963 & 0.405 & 0.287 & 0.899 & -0.151 \\
F2 & 0.871 & 7.287 & 0.951 & -0.129 & $\mathbf{0 . 9 8 6}$ & 23.79 & 0.982 & 0.428 & 0.286 & 0.928 & -0.165 \\
F3 & 0.879 & 8.915 & 0.972 & -0.201 & $\mathbf{0 . 9 8 2}$ & 28.91 & 0.971 & 0.473 & 0.333 & 0.951 & -0.232 \\
F4 & 0.891 & 9.504 & 0.968 & -0.221 & $\mathbf{0 . 9 7 3}$ & 30.46 & 0.941 & 0.509 & 0.305 & 0.951 & -0.254 \\
F5 & 0.909 & 9.858 & $\mathbf{0 . 9 8 4}$ & -0.244 & 0.979 & 31.39 & 0.962 & 0.538 & 0.295 & 0.971 & -0.272 \\
\hline
\end{tabular}




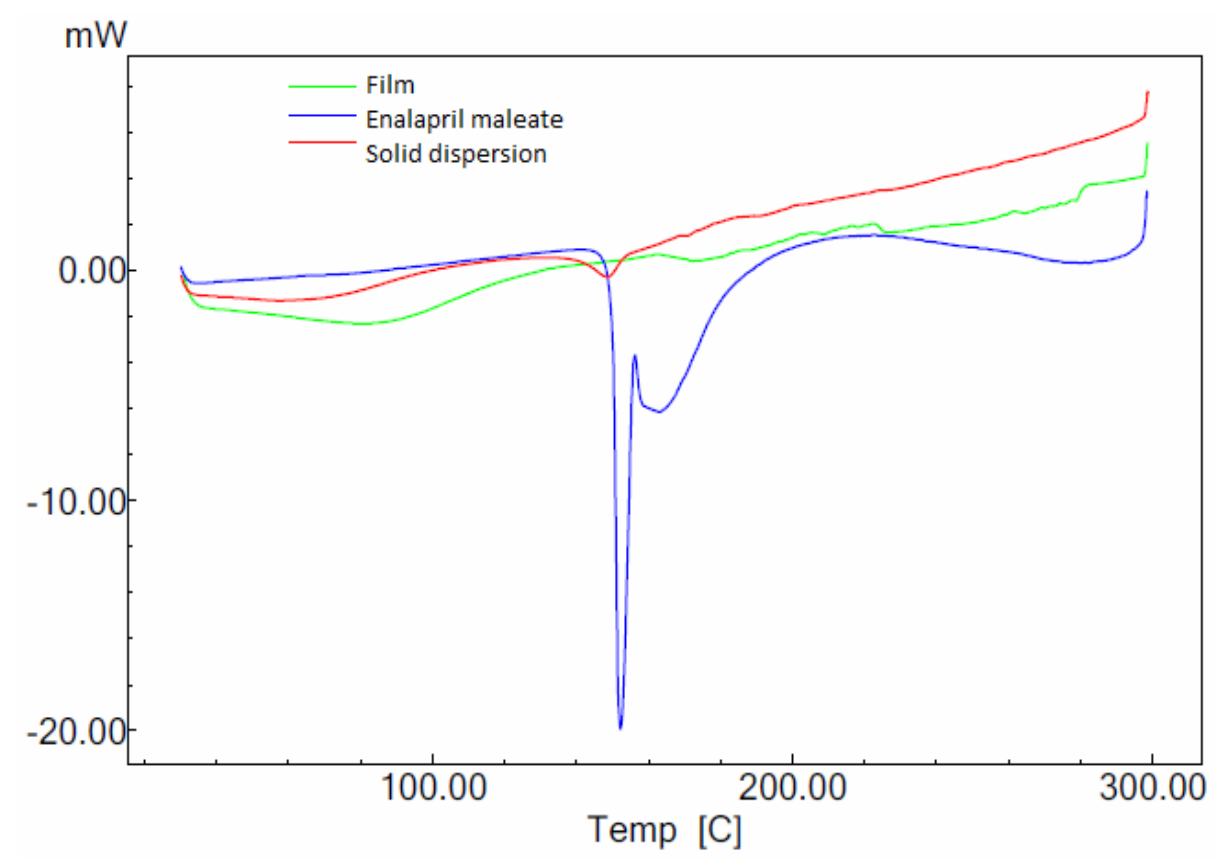

Figure 4. DSC thermograms of pure enalapril maleate, drug loaded film and solid dispersion.
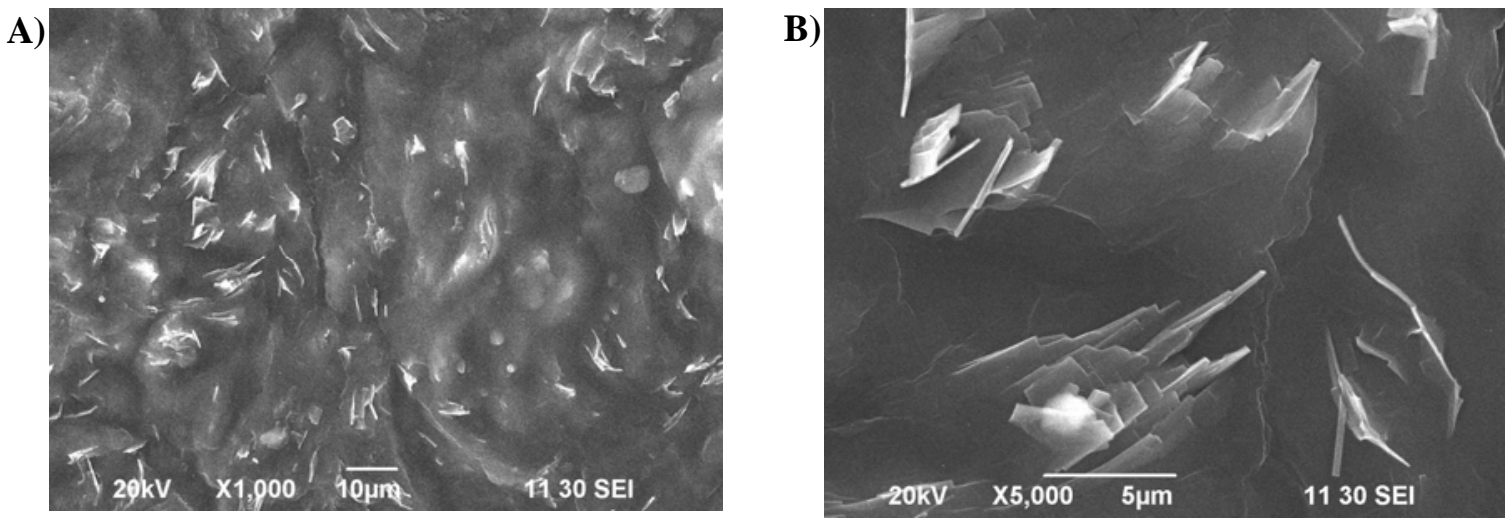

Figure 5. Scanning electron micrographs (A and B) of GRDF of batch F3 at magnification of 1000x and 5000x, respectively.

DSC studies revealed that enalapril maleate exhibits a sharp endothermic peak at $152.42^{\circ} \mathrm{C}$ where onset of melting endotherm at about $150^{\circ} \mathrm{C}$ and endset at about $154^{\circ} \mathrm{C}$ corresponding to its melting point which is usually associated with decomposition of the drug (Figure 4). In the thermogram of solid dispersion, there was a short little bit shifted blund endothermic peak without melting at $148.49^{\circ} \mathrm{C}$ for the drug. This shifting may be due to presence of polymers. This peak did not appear in the thermogram of film which indicated that the drug was uniformly entrapped in the polymeric matrices
Scanning electron microscopy (SEM): The surface morphology of drug loaded polymeric film (batch F3) shown in following figure (Figure 5) at different magnifications (A at a magnification of 1000 and $B$ at a magnification of 5000). The images that were found showed non porous surface and were well integrated. The surface view of the GRDF also showed scattered like crystal structure which may due to deposition of dug or polymers.

Fourier transform infra red (FT-IR) spectroscopy: The FT-IR analysis of batch F3 for drug- polymer mixture (solid dispersion), pure drug (enalapril maleate) and drug loaded gastro retentive 
film (GRDF) showed the spectra A, B and C respectively in Figure 6.

The absorption range (3500-3100) $\mathrm{cm}^{-1}$ in infrared spectroscopy associated with the vibrational mode of primary or secondary amine (-NH). The absorption range $3400-3200 \mathrm{~cm}^{-1}$ represents the presence of $-\mathrm{OH}$ group .The absorption regions in the range of $3300-2500 \mathrm{~cm}^{-1}$ is associated with the presence of $-\mathrm{COOH}$ group. The regions $1750-1730$ $\mathrm{cm}^{-1}$ associated with vibrational mode of ester compound (-COO$\left.{ }^{-}\right)$. The absorption regions 1680$1630 \mathrm{~cm}^{-1}$ represents vibrational mode of amide ($\mathrm{NH}=\mathrm{C}=\mathrm{O}$ ) functional group. The regions (15001450) $\mathrm{cm}^{-1}$ is associated with presence of benzene ring $\left(\mathrm{C}_{6} \mathrm{H}_{6}\right)$.
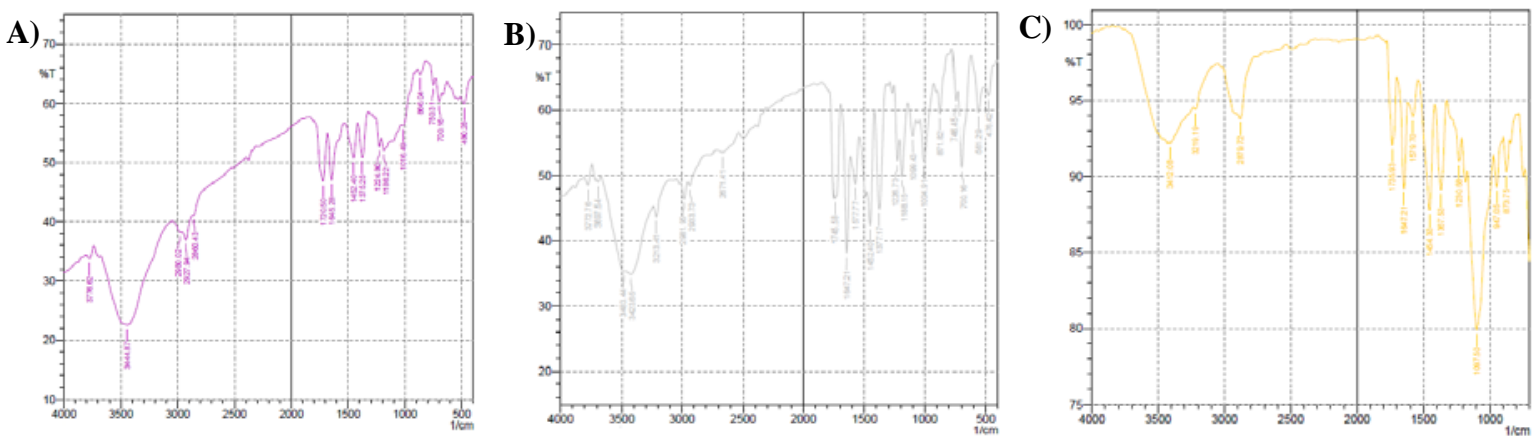

Figure 6. Infrared spectrum of A) drug- polymer physical mixture (solid dispersion), B) Enalapril maleate and C) drug loaded film (GRDF).

The FTIR study revealed that the absorption peaks for the functional groups corresponding to their respective regions were found to be more or less similar for the both IR spectra of solid dispersion of drug-polymer mixture and drug loaded gastro retentive film compared to the spectrum of pure drug enalapril maleate. So it should be concluded that there is no drug polymer interactions in the formulation development of GRDF of enalapril maleate.

\section{Conclusion}

The current research work demonstrated the successful development of an unfolding type expandable gastroretentive dosage form of enalapril maleate for sustained release in the stomach. It comprised of a drug loaded polymeric film of HPMC K15 and eudragit RSPO and eudragit RLPO combination, folded in zigzag pattern and inserted into a hard gelatin capsule. The result revealed that unfolding of the films was satisfactory with folding pattern in simulated gastric fluid. Gastric retention was achieved due to unfolding of the dosage form in the stomach within short time. The GRDFs provided initial loading effect due to fast release of drug from the large surface of the films, later sustained effect where release mechanism involved diffusion through and erosion of the polymeric films. The polymers used in the development of GRDFs were safe and proper combination of these polymers would yield a novel expandable GRDF with good in vitro drug release in acidic media, mechanical properties, and unfolding behavior. We can certainly explore this drug delivery system for further development through its in vivo evaluation studies which may lead to an improved bioavailability and ensured therapy with other already existing drugs of such type.

Unfolding systems might represent one of the best alternatives to resist to the gastric emptying. Indeed the unfolding system is usually introduced through a hard gelatin capsule for an easier intake by the patient. In the stomach the carrier is dissolved due to the action of gastric acid, releasing the GRDF system which unfolds or opens out to achieve its extended configuration. The carrier should maintain unfolded properties for extended time to allow sustained release. This expandable system represents 
an interesting concept and have shown real efficacy but specific attention is needed. Since permanent retention of rigid, large monolithic forms may interfere with gastric motility, causing possible adverse effects such as bowel obstruction, local damage, intestinal adhesion and gastropathy. Finally, this kind of dosage form is probably one of the most difficult to industrialize and might be less cost effective than other forms.

\section{References}

Ali, J., Arora, S. and Khar, R.K. 2005. Floating drug delivery system: A review. AAPS Pharm. Sci. Tech. 6, 372-390.

Banker, G.S., and Anderson, N.R. 2007. Tablets: The Theory and Practice of Industrial Pharmacy. $3^{\text {rd }}$ Edition. Varghese Publishing House, Bombay, p. 293309.

Davies, R.O., Gomez, H.J., Irvin, J.D. and Walker, J.F. 1984. An overview of the clinical pharmacology of enalapril. Br J Clin Pharmacol. 18, 215-229.

Hardman, J.G., Limbird, L.E. and Gilman, A.G. 2001. Goodman and Gilman's The Pharmacological Basis of Therapeutics. 10th ed. New York, NY: McGraw-Hill, p. 823.
Hussar, D.A. 1992. Modell's Drugs in Current Use and New Drugs. 38th ed. New York, NY: Springer Publishing Co., p. 61.

Kagan, L., Lapidotp N. and Afargan, M. 2006. Gastroretentive accordion pill: enhancement of riboflavin bioavailability in humans. J. Cont. Rel. 113, 205-219.

Klausner, E.A., Lavy, E., Friedman, M. and Hofmann, A. 2003. Expandable gastro retentive dosage forms. $J$. Cont. Rel. 90, 143-162.

McEvoy, G.K. 2003. American Hospital Formulary Service- Drug Information. Bethesda, MD: American Society of Health-System Pharmacists, Inc. p. 18501858.

Rouge, N., Buri, P. and Doelker, E. 1996. Drug absorption sites in the gastrointestinal tract and dosage forms for site specific delivery. Int. J. Pharm. 136,117-139.

Singh, B.N., and Kim, K.H. 2000. Floating drug delivery systems: an approach to oral controlled drug delivery via gastric retention. J. Cont. Rel. 63, 235-239.

Streubel, A., Siepmann, J. and Bodmeier, R . 2006. Gastroretentive drug delivery system. Expert Opin. Drug Deliv. 3, 217-233.

Verma, S., Nagpal, K., Singh, S.K. and Mishra, D.N. 2014. Unfolding type gastroretentive film of cinnarizine based on ethyl cellulose and hydroxypropylmethyl cellulose. Int. J. Biol. Macromol. 64, 347-352. 